\title{
A note on a transmission problem for the Brinkman system and the generalized Darcy-Forchheimer-Brinkman system in Lipschitz domains in $\mathbb{R}^{3}$
}

\author{
Andrei-Florin Albişoru
}

\begin{abstract}
The purpose of this paper is to treat a nonlinear transmission-type problem for a generalized version of the Darcy-Forchheimer-Brinkman system and the classical Brinkman system in complementary Lipschitz domains in $\mathbb{R}^{3}$. First of all, we define the required spaces in which we seek our solution. Next, we describe the generalized Brinkman and the generalized Darcy-ForchheimerBrinkman systems. Further, we give important lemmas that allow us to introduce the trace and conormal derivative operators that appear in the formulation of our transmission problem. We invoke a result regarding the well-posedness of the (linear) transmission problem for the generalized and classical Brinkman systems in complementary Lipschitz domains in $\mathbb{R}^{3}$. The above mentioned wellposedness result in the linear case combined with Banach's fixed point theorem will allow us to establish the main result of the paper, the well-posedness of the transmission problem for the Brinkman system and the nonlinear generalized Darcy-Forchheimer-Brinkman system in Lipschitz domains in $\mathbb{R}^{3}$.
\end{abstract}

Mathematics Subject Classification (2010): 35J25, 35Q35, 46E35.

Keywords: Sobolev spaces, generalized Brinkman system, transmission problems, generalized Darcy-Forchheimer-Brinkman system, well-posedness result, Banach fixed point theorem.

\section{Introduction}

Transmission problems that appear in the field of fluid mechanics are important to researchers nowadays, due to their practical applications, such as environmental problems with free air flow interacting with evaporation from soils or transvascular exchange between blood flow in vessel and the surrounding tissue as porous material. Another relevant example is the geophysical model of flow of water or other viscous 
fluids, which pass through porous rocks or porous soil (see [8], [14] and the references therein, and see also [21], [5]). We also mention the important role of the partial differential equations that model different types of flow, such as the Brinkman equations or the Darcy-Forchheimer-Brinkman equations (for additional details, see [21]).

Escauriaza and Mitrea in [4] have established the well-posedness of the transmission problem for the Laplace operator across a Lipschitz interface, for data in Lebesgue and Hardy spaces on the boundary.

Medkova in [17] has studied the transmission problem for the Stokes system with constant coefficients in $\mathbb{R}^{3}$ using the integral equation method.

Mitrea and Wright in [20] have given well-posedness results for transmission problems for the Stokes systems in arbitrary Lipschitz domains in Euclidean setting and in $L^{p}$, Sobolev and Besov spaces.

Groşan, Kohr and Wendland in [6] have studied the Dirichlet problem for the generalized Brinkman system in a bounded Lipschitz domain in $\mathbb{R}^{n}, n \geq 2$ and the Dirichlet problem for the generalized Darcy-Forchheimer-Brinkman system in a bounded Lipschitz domain in $\mathbb{R}^{n}, n=2,3$.

Kohr, Lanza de Cristoforis and Wendland in [13] have treated Poisson problems for a semilinear and a generalized Brinkman system on a bounded Lipschitz domain in $\mathbb{R}^{n}, n \geq 2$, with Dirichlet or Robin boundary conditions and data given in $L^{2}$-based Sobolev spaces.

Kohr, Lanza de Cristoforis and Wendland in [10] have used a layer potential method and a fixed point theorem to show the existence of a solution of the Robin problem for the standard Darcy-Forchheimer-Brinkman system in a bounded Lipschitz domain in $\mathbb{R}^{n}, n=2,3$.

Kohr, Lanza de Cristoforis and Wendland in [9] have studied the Robin problem for the Brinkman and the Darcy-Forchheimer-Brinkman systems with constant coefficients. They also proceeded to study mixed boundary value problems for the Brinkman system and Darcy-Forchheimer-Brinkman system. respectively. Moreover, they have proved a well-posedness result for a boundary problem of mixed DirichletRobin and transmission type for two Brinkman systems.

Medkova in [16] has tackled the transmission problem for the Brinkman system and also the Robin-transmission and the Dirichlet-transmission problems for the Brinkman system in the setting of a bounded Lipschitz domain in $\mathbb{R}^{n}, n>2$. In each of these problems, the systems have constant coefficients.

Kohr, Lanza de Cristoforis and Wendland in [11] have studied nonlinear Neumann-Transmission problems for the (linear) Stokes and Brinkman systems with a nonlinear Neumann condition.

Kohr, Lanza de Cristoforis, Mikhailov and Wendland in [8] have treated transmission problems for the nonlinear Darcy-Forchheimer-Brinkman system and the linear Stokes system in complementary Lipschitz domains in $\mathbb{R}^{3}$.

Kohr, Lanza de Cristoforis and Wendland in [12] have obtained a well-posendess result for the nonlinear Robin-transmission problem for the nonlinear Navier-Stokes and Darcy-Forchheimer-Brinkman systems in the setting of bounded Lipschitz domains in $\mathbb{R}^{n}, n=2,3$. 
Kohr, Mikhailov and Wendland in [14] have obtained well-posedness results for transmission problems for the Navier-Stokes and Darcy-Forchheimer-Brinkman systems in Lipschitz domains on compact Riemannian manifolds of dimension $m=2,3$. The coefficients of these systems of partial differential equations are smooth due to the smoothness of the Riemannian metric tensor.

Mitrea, Mitrea and Shi in [19] have studied variable coefficient transmission problems and singular integral operators on non-smooth manifolds.

Transmission problems for Stokes and Navier-Stokes systems with nonsmooth coefficients $\left(L^{\infty}\right.$-coefficients) on compact Riemannian manifolds have been recently treated by Kohr and Wendland in [15].

The paper is structured as follows. In the second section, we define the Sobolev spaces in which we seek our solutions. There, we describe the generalized versions of the Brinkman system and of the Darcy-Forchheimer-Brinkman system. These systems of PDEs contain $L^{\infty}$ coefficients. We give a result that allows us to consider the trace operator in the setting of Sobolev spaces (Lemma 2.5). In addition, we mention a result that allows us to consider the conormal derivative operator for the generalized Brinkman system (Lemma 2.6). We end this section with two results. The first of them is related to the growth conditions at infinity of a pair $(\mathbf{w}, r)$ that satisfy the homogeneous Brinkman equation with constant coefficients in an exterior Lipschitz domain in $\mathbb{R}^{3}$ (Lemma 2.7). The second result gives mapping properties of a nonlinear operator related with our nonlinear transmission problem (Lemma 2.8). In the third section, we state the well-posedness result for the linear transmission problem for the classical and generalized Brinkman systems in complementary Lipschitz domains in $\mathbb{R}^{3}$ (Theorem 3.1). Using this well-posedness result and the Banach fixed point theorem, we obtain the well-posedness result for the main nonlinear problem considered in this paper, which is the transmission problem for the Brinkman and the generalized DarcyForchheimer-Brinkman systems in complementary Lipschitz domains in $\mathbb{R}^{3}$, namely Theorem 3.2.

\section{Preliminaries}

In this paper, by the superscript ' we refer to the topological dual of a given space. Also, we use the notation $\langle\cdot, \cdot\rangle_{A}$ to denote the duality pairing of two dual Sobolev spaces defined on $A$, where $A$ is either an open set or a surface in $\mathbb{R}^{3}$. Also, denote by $\mathrm{E}(\mathbf{w})$ the symmetric part of $\nabla \mathbf{w}$ (where $\mathbf{w}$ is a given field),

$$
\mathrm{E}(\mathbf{w}):=\frac{1}{2}\left(\nabla \mathbf{w}+\nabla \mathbf{w}^{t}\right)
$$

and the superscript $t$ refers to the transpose. Also, by $\stackrel{\circ}{E}$, we denote the operator of extension by zero outside our considered bounded Lipschitz domain $\Omega \subset \mathbb{R}^{3}$.

Next, we introduce the Sobolev spaces in which we seek the solution of our transmission problem. Also, we describe the generalized version of the Darcy-ForchheimerBrinkman system and we give the results that allow us to introduce the trace and conormal derivative operators, operators that appear in the boundary conditions of our problem. 
To this end, let $\Omega_{+}:=\Omega \subset \mathbb{R}^{3}$ be a bounded Lipschitz domain (an open, connected set, whose boundary is locally the graph of a Lipschitz function) with connected boundary (denoted by $\partial \Omega$ ) and by $\Omega_{-}:=\mathbb{R}^{3} \backslash \bar{\Omega}$, we denote the corresponding complementary Lipschitz set.

In the latter $\Omega_{0}$ denotes either one of the following sets: $\Omega_{+}, \Omega_{-}$or $\mathbb{R}^{3}$.

Recall that $\mathcal{D}\left(\Omega_{0}\right)$ is the space of compactly supported smooth functions $C_{0}^{\infty}\left(\Omega_{0}\right)$ and by $\mathcal{D}^{\prime}\left(\Omega_{0}\right)$ we denote its dual, the space of distributions on $\Omega_{0}$. Note that $\mathcal{D}\left(\Omega_{0}\right)$ is endowed with the inductive limit topology and $\mathcal{D}^{\prime}\left(\Omega_{0}\right)$ is endowed with the weak-star topology.

Definition 2.1. Let $p \in[1, \infty)$. Then, the Lebesgue space $L^{p}\left(\mathbb{R}^{3}\right)$ is the space of all (equivalence classes of) measurable functions $f: \mathbb{R}^{3} \rightarrow \mathbb{R}$ with the property that:

$$
\int_{\mathbb{R}^{3}}|f(x)|^{p} \mathrm{~d} x<\infty .
$$

We also denote by $\mathcal{F}$ the Fourier transform and by $\mathcal{F}^{-1}$ its inverse acting on functions from $L^{1}\left(\mathbb{R}^{3}\right)$. We shall consider their generalization to the space of tempered distributions.

We have the following definition (see, e.g., [8, (2.1)-(2.3)], [1, Ch. 1], [7, Ch. 4]).

Definition 2.2. Let $s \in \mathbb{R}$. Introduce the $L^{2}$-based (Bessel potential) Sobolev spaces by:

$$
\begin{aligned}
& H^{s}\left(\mathbb{R}^{3}\right):=\left\{\mathcal{F}^{-1}\left(1+|\xi|^{2}\right)^{-\frac{s}{2}} \mathcal{F} u: u \in L^{2}\left(\mathbb{R}^{3}\right)\right\}, \\
& H^{s}\left(\Omega_{0}\right):=\left\{u \in \mathcal{D}^{\prime}\left(\Omega_{0}\right): \exists U \in H^{s}\left(\mathbb{R}^{3}\right) \text { such that }\left.U\right|_{\Omega_{0}}=u\right\}, \\
& \widetilde{H}^{s}\left(\Omega_{0}\right):=\overline{\mathcal{D}\left(\Omega_{0}\right)}|| \cdot \|_{H^{s}\left(\mathbb{R}^{3}\right)},
\end{aligned}
$$

hence $\widetilde{H}^{s}\left(\Omega_{0}\right)$ is the closure of $\mathcal{D}\left(\Omega_{0}\right)$ in $H^{s}\left(\mathbb{R}^{3}\right)$.

One may also introduce the vector-valued spaces component-wise.

We also have the following definition (see also [7, p. 169]).

Definition 2.3. Let $s \in(0,1)$. Then, the boundary Sobolev spaces $H^{s}(\partial \Omega)$ is defined by:

$$
\begin{aligned}
H^{s}(\partial \Omega):=\left\{u \in L^{2}(\partial \Omega):\|u\|_{H^{s}(\partial \Omega)}=\|u\|_{L^{2}(\partial \Omega)}+\right. \\
\left.\qquad \int_{\partial \Omega} \int_{\partial \Omega} \frac{|u(x)-u(y)|}{|x-y|^{2+2 s}} d \sigma_{x} d \sigma_{y}<\infty\right\} .
\end{aligned}
$$

The following duality

$$
H^{-s}(\partial \Omega):=\left(H^{s}(\partial \Omega)\right)^{\prime},
$$

allows us to introduce the boundary Sobolev space with negative order $H^{-s}(\partial \Omega)$. Again, the vector-valued spaces are introduced component-wise. Note that, all these $L^{2}$-based Sobolev spaces are Hilbert spaces (see, e.g., [1]).

We also describe here the space $\mathfrak{M}\left(\Omega_{0}\right)$ (see $[8$, p. 23$]$ ), i.e., the space in which we shall seek the unknown pressure field in the exterior Lipschitz domain in our transmission problem. 
Let us consider the weight function:

$$
\rho(\mathbf{x}):=\left(1+|\mathbf{x}|^{2}\right)^{\frac{1}{2}}, \forall x \in \mathbb{R}^{3} .
$$

Then, the weighted Lebesgue space $L^{2}\left(\rho^{-1} ; \Omega_{0}\right)$ is the set of all functions $v$ with the property that $\rho^{-1} u \in L^{2}\left(\Omega_{0}\right)$.

Moreover, the space $\mathfrak{M}\left(\Omega_{0}\right)$ is defined by:

$$
\mathfrak{M}\left(\Omega_{0}\right):=\left\{\mathfrak{g} \in L^{2}\left(\rho^{-1}, \Omega_{0}\right): \nabla \mathfrak{g} \in H_{\text {curl }}^{-1}\left(\Omega_{0}\right)^{3}\right\},
$$

where, by $H_{\text {curl }}^{-1}\left(\Omega_{0}\right)^{3}$ we understand the space:

$$
H_{\text {curl }}^{-1}\left(\Omega_{0}\right)^{3}:=\left\{\mathfrak{h} \in H^{-1}\left(\Omega_{0}\right)^{3}: \operatorname{curl} \mathfrak{h}=\nabla \times \mathfrak{h}=0\right\} .
$$

By denoting $\mathfrak{M}^{\prime}\left(\Omega_{0}\right)$ the dual of $\mathfrak{M}\left(\Omega_{0}\right)$, we have the very suggestive chain of continuous embeddings (cf. [8, (A.24)]):

$$
L^{2}\left(\rho, \Omega_{0}\right) \subset \mathfrak{M}^{\prime}\left(\Omega_{0}\right) \subset L^{2}\left(\Omega_{0}\right) \subset \mathfrak{M}\left(\Omega_{0}\right) \subset L^{2}\left(\rho^{-1}, \Omega_{0}\right) \subset L_{\mathrm{loc}}^{2}\left(\Omega_{0}\right) .
$$

The generalized version of the Brinkman system of PDEs, is given by (see, e.g., $[13$, Relation (2.14)]):

$$
\Delta \mathbf{w}-\mathcal{P} \mathbf{w}-\nabla p=\mathfrak{F} \text { in } \Omega_{+}, \operatorname{div} \mathbf{w}=0 \text { in } \Omega_{+},
$$

where $\mathcal{P} \in L^{\infty}\left(\Omega_{+}\right)^{3 \times 3}$ satisfies the condition:

$$
\langle\mathcal{P} \mathbf{v}, \mathbf{v}\rangle_{\Omega_{+}} \geq c_{\mathcal{P}}\|\mathbf{v}\|_{L^{2}\left(\Omega_{+}\right)^{3}}^{2}, \forall \mathbf{v} \in L^{2}\left(\Omega_{+}\right)^{3},
$$

with some constant $c_{\mathcal{P}}>0$ is a constant.

The important generalization that we consider in this work is the generalized version of the Darcy-Forchheimer-Brinkman system:

$$
\Delta \mathbf{w}-\mathcal{P} \mathbf{w}-k|\mathbf{w}| \mathbf{w}-\beta(\mathbf{w} \cdot \nabla) \mathbf{w}-\nabla p=\mathfrak{F} \text { in } \Omega_{+}, \operatorname{div} \mathbf{w}=0 \text { in } \Omega_{+},
$$

with $\mathcal{P} \in L^{\infty}\left(\Omega_{+}\right)^{3 \times 3}$ as above, $k, \beta: \Omega_{+} \rightarrow \mathbb{R}_{+}$are functions such that $k, \beta \in$ $L^{\infty}\left(\Omega_{+}\right)$, i.e., essentially bounded, non-negative functions defined on $\Omega_{+}$.

Remark 2.4. (i) If we let $\mathcal{P}=\alpha \mathbb{I}$ where $\alpha>0$ is a constant in (2.1), we get the classical Brinkman system.

(ii) If $\mathcal{P}=0$ in (2.1), we get the well-known Stokes system.

(iii) If $\mathcal{P} \equiv \alpha \mathbb{I}$, where $\alpha>0$ is a constant and $k, \beta>0$ are also constants in (2.3), one obtains the classical Darcy-Forchheimer-Brinkman system.

(iv) If $\mathcal{P} \equiv 0, k=0$ and $\beta>0$ is a constant in (2.3), we recover the Navier-Stokes system.

Next, we introduce the following result that allows us to define the trace operator (see, e.g., [18, Theorem 2.3]).

Lemma 2.5. (Gagliardo Trace Lemma) Let $\Omega_{+}:=\Omega \subset \mathbb{R}^{3}$ be a bounded Lipschitz domain with connected boundary $\partial \Omega$ and denote by $\Omega_{-}:=\mathbb{R}^{3} \backslash \bar{\Omega}$ the complementary Lipschitz set. Then, there exist linear, continuous trace operators $\operatorname{Tr}^{ \pm}: H^{1}\left(\Omega_{ \pm}\right) \rightarrow$ $H^{\frac{1}{2}}(\partial \Omega)$, such that

$$
\operatorname{Tr}^{ \pm} u=\left.u\right|_{\partial \Omega}, \quad \forall v \in C^{\infty}\left(\overline{\Omega_{ \pm}}\right) .
$$

Moreover, these operators are surjective, having (non-unique) linear and continuous right inverse operators $Z^{ \pm}: H^{\frac{1}{2}}(\partial \Omega) \rightarrow H^{1}\left(\Omega_{ \pm}\right)$. 
We have the following result that allows us to consider the conormal derivative for the generalized Brinkman system (see, e.g., [13, Lemma 2.3]).

Lemma 2.6. Let $\Omega_{+}:=\Omega \subset \mathbb{R}^{3}$, be a bounded Lipschitz domain with connected boundary $\partial \Omega$. Let $\mathcal{P} \in L^{\infty}\left(\Omega_{+}\right)^{3 \times 3}$. Consider the following space:

$$
\begin{aligned}
\boldsymbol{H}^{1}\left(\Omega_{+}, \mathcal{B}_{\mathcal{P}}\right):= & \left\{(\mathbf{w}, p, \mathfrak{F}) \in H^{1}\left(\Omega_{+}\right)^{3} \times L^{2}\left(\Omega_{+}\right) \times \widetilde{H}^{-1}\left(\Omega_{+}\right)^{3}:\right. \\
& \mathcal{B}_{\mathcal{P}}(\mathbf{w}, p):=\Delta w-\mathcal{P} \mathbf{w}-\nabla p=\left.\mathfrak{F}\right|_{\Omega_{+}} \\
& \text {and } \left.\operatorname{div} \mathbf{w}=0 \text { in } \Omega_{+}\right\} .
\end{aligned}
$$

Define the conormal derivative operator for the generalized Brinkman system,

$$
\mathbf{t}_{\mathcal{P}, \nu}^{+}: \boldsymbol{H}^{1}\left(\Omega_{+}, \mathcal{B}_{\mathcal{P}}\right) \rightarrow H^{-\frac{1}{2}}(\partial \Omega)^{3},
$$

by the following relation:

$$
\begin{aligned}
\left\langle\mathbf{t}_{\mathcal{P}, \nu}^{+}(\mathbf{w}, p, \mathfrak{F}), \boldsymbol{\phi}\right\rangle_{\partial \Omega}:= & 2\left\langle\mathrm{E}(\mathbf{w}), \mathrm{E}\left(Z^{+} \boldsymbol{\phi}\right)\right\rangle_{\Omega_{+}}+\left\langle\mathcal{P} \mathbf{w}, Z^{+} \boldsymbol{\phi}\right\rangle_{\Omega_{+}} \\
& -\left\langle p, \operatorname{div}\left(Z^{+} \boldsymbol{\phi}\right)\right\rangle_{\Omega_{+}} \\
& +\left\langle\mathfrak{F}, Z^{+} \boldsymbol{\phi}\right\rangle_{\Omega_{+}}, \quad \forall \boldsymbol{\phi} \in H^{-\frac{1}{2}}(\partial \Omega)^{3},
\end{aligned}
$$

where $Z^{+}$is a right inverse of the trace operator $\operatorname{Tr}^{+}: H^{1}\left(\Omega_{+}\right)^{3} \rightarrow H^{\frac{1}{2}}(\partial \Omega)^{3}$. The operator $\mathbf{t}_{\mathcal{P}, \nu}^{+}$is linear, bounded and does not depend on the choice of the right inverse $Z^{+}$of the trace operator $\mathrm{Tr}^{+}$.

Moreover, the following Green formula holds:

$$
\begin{aligned}
\left\langle\mathbf{t}_{\mathcal{P}, \nu}^{+}(\mathbf{w}, p, \mathfrak{F}), \operatorname{Tr}^{+} \boldsymbol{\psi}\right\rangle_{\partial \Omega}= & 2\langle\mathrm{E}(\mathbf{w}), \mathrm{E}(\boldsymbol{\psi})\rangle_{\Omega_{+}}+\langle\mathcal{P} \mathbf{w}, \boldsymbol{\psi}\rangle_{\Omega_{+}} \\
& -\langle p, \operatorname{div} \boldsymbol{\psi}\rangle_{\Omega_{+}}+\langle\mathfrak{F}, \boldsymbol{\psi}\rangle_{\Omega_{+}},
\end{aligned}
$$

for $\operatorname{all}(\mathbf{w}, p, \mathfrak{F}) \in \boldsymbol{H}^{1}\left(\Omega_{+}, \mathcal{B}_{\mathcal{P}}\right)$ and for any $\boldsymbol{\psi} \in H^{1}\left(\Omega_{+}\right)^{3}$.

Similarly, one may introduce the conormal derivative operator for the classical Brinkman system which is denoted by $\mathbf{t}_{\alpha, \nu}^{ \pm}$where $\alpha>0$ is a constant. The statement of the lemma for the introduction of the above described operator is omitted for the sake of brevity, but we refer the reader to [8, Lemma 2.5].

Next, we are concerned with the behavior at infinity of a solution of the classical homogeneous Brinkman system in the unbounded domain $\Omega_{-}$. We have the following lemma (cf. [2, Lemma A.2]).

Lemma 2.7. Let $\alpha>0$ be a constant. Let $\Omega_{+}:=\Omega \subset \mathbb{R}^{3}$ be a bounded Lipschitz domain with connected boundary and let $\Omega_{-}:=\mathbb{R}^{3} \backslash \bar{\Omega}$. If the pair $(\mathbf{w}, r) \in H^{1}\left(\Omega_{-}\right)^{3} \times \mathfrak{M}\left(\Omega_{-}\right)$ satisfy the Brinkman equations:

$$
\Delta \mathbf{w}-\alpha \mathbf{w}-\nabla r=0, \quad \operatorname{div} \mathbf{w}=0, \quad \text { in } \Omega_{-},
$$

then

$$
\mathbf{w}(\mathbf{x})=O\left(|\mathbf{x}|^{-2}\right), \quad \nabla \mathbf{w}(\mathbf{x})=O\left(|\mathbf{x}|^{-1}\right), \quad r(\mathbf{x})=O\left(|\mathbf{x}|^{-1}\right), \quad \text { as }|\mathbf{x}| \rightarrow \infty .
$$

The proof of this lemma can be consulted in [2]. 
Finally, we mention a lemma that gives an important characterization of the following nonlinear operator that appears in the nonlinear transmission problem:

$$
\mathcal{J}_{k, \beta, \Omega_{+}}(\mathbf{v}):=\stackrel{\circ}{E}(k|\mathbf{v}| \mathbf{v}+\beta(\mathbf{v} \cdot \nabla) \mathbf{v}) .
$$

The mapping and other properties of this operator are provided below (see, e.g., $[3$, Lemma 3.1] and [8, Lemma 5.1] in the case $\mathcal{P}=\alpha \mathbb{I}$, where $\alpha>0$ is a constant and $k, \beta>0$ are constants).

Lemma 2.8. Let $\Omega_{+}:=\Omega \subset \mathbb{R}^{3}$ be a bounded Lipschitz domain with connected boundary. Let $k, \beta: \Omega_{+} \rightarrow \mathbb{R}_{+}$such that $k, \beta \in L^{\infty}\left(\Omega_{+}\right)$and let

$$
\mathcal{J}_{k, \beta, \Omega_{+}}(\mathbf{v}):=\stackrel{\circ}{E}(k|\mathbf{v}| \mathbf{v}+\beta(\mathbf{v} \cdot \nabla) \mathbf{v}) .
$$

Then, the nonlinear operator $\mathcal{J}_{k, \beta, \Omega_{+}}: H_{\mathrm{div}}^{1}\left(\Omega_{+}\right)^{3} \rightarrow \widetilde{H}^{-1}\left(\Omega_{+}\right)^{3}$ is continuous, positively homogeneous of order 2 , and bounded, in the sense that there is a constant $c_{0}=c_{0}\left(\Omega_{+}, k, \beta\right)>0$ such that

$$
\left\|\mathcal{J}_{k, \beta, \Omega_{+}}(\mathbf{v})\right\|_{\widetilde{H}^{-1}\left(\Omega_{+}\right)^{3}} \leq c_{0}\|\mathbf{v}\|_{H^{1}\left(\Omega_{+}\right)^{3}}^{2} .
$$

Moreover, the following Lipschitz-like relation holds:

$$
\begin{aligned}
\| \mathcal{J}_{k, \beta, \Omega_{+}}(\mathbf{v}) & -\mathcal{J}_{k, \beta, \Omega_{+}}(\mathbf{w}) \|_{\widetilde{H}^{-1}\left(\Omega_{+}\right)^{3}} \\
& \leq c_{0}\left(\|\mathbf{v}\|_{H^{1}\left(\Omega_{+}\right)^{3}}+\|\mathbf{w}\|_{H^{1}\left(\Omega_{+}\right)^{3}}\right)\|\mathbf{v}-\mathbf{w}\|_{H^{1}\left(\Omega_{+}\right)^{3}},
\end{aligned}
$$

with $c_{0}=c_{0}\left(\Omega_{+}, k, \beta\right)>0$ is the same constant as in relation $(2.11)$.

Proof. We provide here the main ideas that lead to the statement of the lemma (for additional details, see the proof of Lemma 5.1 in [8]).

First, we have the following continuous embeddings, due to the Sobolev embedding theorem (see, e.g., [7, Theorem 4.1.5, Theorem 4.1.6]):

$$
H^{1}\left(\Omega_{+}\right)^{3} \hookrightarrow L^{q}\left(\Omega_{+}\right)^{3}, \quad L^{q^{\prime}}\left(\Omega_{+}\right)^{3} \hookrightarrow \widetilde{H}^{-1}\left(\Omega_{+}\right)^{3},
$$

where $2 \leq q \leq 6$, and $\frac{1}{q}+\frac{1}{q^{\prime}}=1$.

Using relations (2.13) and Hölder's inequality, one may show that $|\mathbf{v}| \mathbf{w} \in$ $L^{2}\left(\Omega_{+}\right)^{3}$ and $(\mathbf{v} \cdot \nabla) \mathbf{w} \in L^{\frac{3}{2}}\left(\Omega_{+}\right)^{3}$, for all $\mathbf{v}, \mathbf{w} \in H^{1}\left(\Omega_{+}\right)^{3}$.

Let us now consider the operators

$$
\begin{aligned}
& b_{1}: H^{1}\left(\Omega_{+}\right)^{3} \times H^{1}\left(\Omega_{+}\right)^{3} \rightarrow \widetilde{H}^{-1}\left(\Omega_{+}\right)^{3}, \\
& b_{2}: H^{1}\left(\Omega_{+}\right)^{3} \times H^{1}\left(\Omega_{+}\right)^{3} \rightarrow \widetilde{H}^{-1}\left(\Omega_{+}\right)^{3},
\end{aligned}
$$

given by

$$
\begin{aligned}
& b_{1}(\mathbf{v}, \mathbf{w}):=\stackrel{\circ}{E}(k|\mathbf{v}| \mathbf{w}), \\
& b_{2}(\mathbf{v}, \mathbf{w}):=\stackrel{\circ}{E}(\beta(\mathbf{v} \cdot \nabla) \mathbf{w}) .
\end{aligned}
$$

Using the embeddings (2.13) and again Hölder's inequality, one may show that there are two constants $c_{*}=c_{*}\left(\Omega_{+}, k\right)>0$ and $c^{*}=c^{*}\left(\Omega_{+}, \beta\right)>0$ such that the following relations hold:

$$
\begin{aligned}
\left\|b_{1}(\mathbf{v}, \mathbf{w})\right\|_{\widetilde{H}^{-1}\left(\Omega_{+}\right)^{3}} \leq c_{*}\|\mathbf{v}\|_{H^{1}\left(\Omega_{+}\right)^{3}}\|\mathbf{w}\|_{H^{1}\left(\Omega_{+}\right)^{3}}, \\
\left\|b_{2}(\mathbf{v}, \mathbf{w})\right\|_{\widetilde{H}^{-1}\left(\Omega_{+}\right)^{3}} \leq c^{*}\|\mathbf{v}\|_{H^{1}\left(\Omega_{+}\right)^{3}}\|\mathbf{w}\|_{H^{1}\left(\Omega_{+}\right)^{3}},
\end{aligned}
$$


which show the continuity of the operators $b_{1}$ and $b_{2}$.

Note that, the operator $\mathcal{J}_{k, \beta, \Omega_{+}}$can be written as:

$$
\mathcal{J}_{k, \beta, \Omega_{+}}(\mathbf{v})=b_{1}(\mathbf{v}, \mathbf{v})+b_{2}(\mathbf{v}, \mathbf{v}),
$$

and by employing the relations (2.16), we have that $\mathcal{J}_{k, \beta, \Omega_{+}}$satisfies (2.11) with $c_{0}=c_{*}+c^{*}$, as asserted.

Also, by using similar arguments to those in the proof of [8, Lemma 5.1] and again relations $(2.16)$, one shows that the operator $\mathcal{J}_{k, \beta, \Omega_{+}}$satisfies the Lipschitz-like condition (2.12).

The full argument is omitted for the sake of brevity.

\section{The main result}

We introduce the following spaces:

$$
\begin{aligned}
& H_{\text {div }}^{1}\left(\Omega_{+}\right)^{3}:=\left\{\mathbf{w} \in H^{1}\left(\Omega_{+}\right)^{3}: \operatorname{div} \mathbf{w}=0 \text { in } \Omega_{+}\right\}, \\
& H_{\text {div }}^{1}\left(\Omega_{-}\right)^{3}:=\left\{\mathbf{w} \in H^{1}\left(\Omega_{-}\right)^{3}: \operatorname{div} \mathbf{w}=0 \text { in } \Omega_{-}\right\}, \\
& \mathfrak{X}:=H_{\text {div }}^{1}\left(\Omega_{+}\right)^{3} \times L^{2}\left(\Omega_{+}\right) \times H_{\text {div }}^{1}\left(\Omega_{-}\right)^{3} \times L^{2}\left(\Omega_{-}\right), \\
& \mathfrak{Y}:=\widetilde{H}^{-1}\left(\Omega_{+}\right)^{3} \times \widetilde{H}^{-1}\left(\Omega_{-}\right)^{3} \times H^{\frac{1}{2}}(\partial \Omega)^{3} \times H^{-\frac{1}{2}}(\partial \Omega)^{3} .
\end{aligned}
$$

Let $\boldsymbol{L} \in L^{\infty}(\partial \Omega)^{3 \times 3}$ be a symmetric matrix-valued function, which satisfies the following positivity condition:

$$
\langle\boldsymbol{L} \mathbf{v}, \mathbf{v}\rangle_{\partial \Omega} \geq 0, \quad \forall \mathbf{v} \in L^{2}(\partial \Omega)^{3} .
$$

Before we state the main result of this paper, we invoke an auxiliary property which refers to the well-posedness result of the Poisson problem of transmission-type for the generalized Brinkman system and classical Brinkman system in complementary Lipschitz domains in $\mathbb{R}^{3}$ and in the space $\mathfrak{X}$. Such a result is useful to obtain the existence of a solution (and its uniqueness) in the space $\mathfrak{X}$ for the nonlinear transmission problem concerning the classical Brinkman and the generalized DarcyForchheimer-Brinkman systems in complementary Lipschitz domains in $\mathbb{R}^{3}$.

The result is as follows (cf. [2, Theorem 3.3]).

Theorem 3.1. Let $\alpha>0$ be a given constant. Let $\Omega_{+}:=\Omega \subset \mathbb{R}^{3}$ be a bounded Lipschitz domain with connected boundary and let $\Omega_{-}:=\mathbb{R}^{3} \backslash \bar{\Omega}$ the complementary Lipschitz set. Let $\mathcal{P} \in L^{\infty}\left(\Omega_{+}\right)^{3 \times 3}$ be such that condition $(2.2)$ holds. Let $\boldsymbol{L} \in L^{\infty}(\partial \Omega)^{3 \times 3}$ be a symmetric matrix-valued function that satisfied condition (3.1). Then, for given data $\left(\mathfrak{F}_{+}, \mathfrak{F}_{-}, \mathfrak{G}_{0}, \mathfrak{H}_{0}\right) \in \mathfrak{Y}$, the Poisson problem of transmission-type for the classical Brinkman and the generalized Brinkman systems:

$$
\left\{\begin{array}{l}
\Delta \mathbf{w}_{+}-\mathcal{P}_{\mathbf{w}_{+}}-\nabla p_{+}=\left.\mathfrak{F}\right|_{\Omega_{+}} \text {in } \Omega_{+}, \\
\Delta \mathbf{w}_{-}-\alpha \mathbf{w}_{-}-\nabla p_{-}=\left.\mathfrak{F}\right|_{\Omega_{-}} \text {in } \Omega_{-}, \\
\operatorname{Tr}^{+} \mathbf{w}_{+}-T r^{-} \mathbf{w}_{-}=\mathfrak{G}_{0} \text { on } \partial \Omega, \\
\mathbf{t}_{\mathcal{P}_{, \nu}}^{+}\left(\mathbf{w}_{+}, p_{+}, \mathfrak{F}_{+}\right)-\mathbf{t}_{\alpha, \nu}^{-}\left(\mathbf{w}_{-}, p_{-}, \mathfrak{F}_{-}\right)+\boldsymbol{L} T r^{+} \mathbf{w}_{+}=\mathfrak{H}_{0} \text { on } \partial \Omega,
\end{array}\right.
$$


has a unique solution $\left(\mathbf{w}_{+}, p_{+}, \mathbf{w}_{-}, p_{-}\right) \in \mathfrak{X}$. In addition, the 'solution' operator:

$$
\mathcal{T}: \mathfrak{Y} \rightarrow \mathfrak{X},
$$

that maps the given data $\left(\mathfrak{F}_{+}, \mathfrak{F}_{-}, \mathfrak{G}_{0}, \mathfrak{H}_{0}\right) \in \mathfrak{Y}$ to the solution $\left(\mathbf{w}_{+}, p_{+}, \mathbf{w}_{-}, p_{-}\right) \in \mathfrak{X}$ of the transmission problem (3.2), is well-defined, linear and continuous.

Hence, there is a constant $C \equiv C\left(\Omega_{+}, \Omega_{-}, \mathcal{P}, \boldsymbol{L}\right)>0$ such that:

$$
\left\|\left(\mathbf{w}_{+}, p_{+}, \mathbf{w}_{-}, p_{-}\right)\right\|_{\mathfrak{X}} \leq C\left\|\left(\mathfrak{F}_{+}, \mathfrak{F}_{-}, \mathfrak{G}_{0}, \mathfrak{H}_{0}\right)\right\|_{\mathfrak{Y}} .
$$

The proof of this result can be consulted in [2].

The main result of this paper which is the well-posedness result for the transmission problem for the generalized Darcy-Forchheimer-Brinkman system and classical Brinkman system in complementary Lipschitz domains in $\mathbb{R}^{3}$. We aim to determine the unknown fields $\left(\mathbf{w}_{+}, p_{+}, \mathbf{w}_{-}, p_{-}\right) \in \mathfrak{X}$ such that:

$$
\left\{\begin{array}{l}
\Delta \mathbf{w}_{+}-\mathcal{P} \mathbf{w}_{+}-k\left|\mathbf{w}_{+}\right| \mathbf{w}_{+}-\beta\left(\mathbf{w}_{+} \cdot \nabla\right) \mathbf{w}_{+} \\
\quad-\nabla p_{+}=\left.\mathfrak{F}\right|_{\Omega_{+}} \text {in } \Omega_{+}, \\
\Delta \mathbf{w}_{-}-\alpha \mathbf{w}_{-}-\nabla p_{-}=\left.\mathfrak{F}\right|_{\Omega_{-}} \text {in } \Omega_{-}, \\
T r^{+} \mathbf{w}_{+}-\operatorname{Tr}^{-} \mathbf{w}_{-}=\mathfrak{G}_{0} \text { on } \partial \Omega \\
\mathbf{t}_{\mathcal{P}, \nu}^{+}\left(\mathbf{w}_{+}, p_{+}, \mathfrak{F}_{+}+\stackrel{\circ}{E}\left(k\left|\mathbf{w}_{+}\right| \mathbf{w}_{+}+\beta\left(\mathbf{w}_{+} \cdot \nabla\right) \mathbf{w}_{+}\right)\right) \\
\quad-\mathbf{t}_{\alpha, \nu}^{-}\left(\mathbf{w}_{-}, p_{-}, \mathfrak{F}_{-}\right)+\boldsymbol{L} \operatorname{Tr}^{+} \mathbf{w}_{+}=\mathfrak{H}_{0} \text { on } \partial \Omega,
\end{array}\right.
$$

where $\alpha>0$ is a given constant.

The main result of the paper reads as follows (see e.g., [3, Theorem 3.2], and [8, Theorem 5.2] in the case $\mathcal{P}=\alpha \mathbb{I}$, where $\alpha, k, \beta>0$ are constants).

Theorem 3.2. Let $\alpha>0$ be a given constant. Let $\Omega_{+}:=\Omega \subset \mathbb{R}^{3}$ be a bounded Lipschitz domain with connected boundary and let $\Omega_{-}:=\mathbb{R}^{3} \backslash \bar{\Omega}$ the complementary Lipschitz set. Let $\mathcal{P} \in L^{\infty}\left(\Omega_{+}\right)^{3 \times 3}$ be such that condition $(2.2)$ holds. Let $\boldsymbol{L} \in L^{\infty}(\partial \Omega)^{3 \times 3}$ be a symmetric matrix-valued function that satisfies condition (3.1). Then, there exist two constants $\xi=\xi\left(\Omega_{+}, \Omega_{-}, \mathcal{P}, k, \beta, \boldsymbol{L}\right)>0$ and $\lambda=\lambda\left(\Omega_{+}, \Omega_{-}, \mathcal{P}, k, \beta, \boldsymbol{L}\right)>0$, such that for all given data $\left(\mathfrak{F}_{+}, \mathfrak{F}_{-}, \mathfrak{G}_{0}, \mathfrak{H}_{0}\right) \in \mathfrak{Y}$ that satisfy the condition

$$
\left\|\left(\mathfrak{F}_{+}, \mathfrak{F}_{-}, \mathfrak{G}_{0}, \mathfrak{H}_{0}\right)\right\|_{\mathfrak{Y}} \leq \xi
$$

the transmission problem (3.5) has a unique solution $\left(\mathbf{w}_{+}, p_{+}, \mathbf{w}_{-}, p_{-}\right) \in \mathfrak{X}$ such that

$$
\left\|\mathbf{w}_{+}\right\|_{H_{\mathrm{div}}^{1}\left(\Omega_{+}\right)^{3}} \leq \lambda .
$$

In addition, the solution depends continuously on the given data, which means that there exists a given constant $C_{0}=C_{0}\left(\Omega_{+}, \Omega_{-}, \mathcal{P}, \boldsymbol{L}\right)>0$ such that:

$$
\left\|\left(\mathbf{w}_{+}, p_{+}, \mathbf{w}_{-}, p_{-}\right)\right\|_{\mathfrak{X}} \leq C_{0}\left\|\left(\mathfrak{F}_{+}, \mathfrak{F}_{-}, \mathfrak{G}_{0}, \mathfrak{H}_{0}\right)\right\|_{\mathfrak{Y}} .
$$

Proof. We provide here only the main ideas of the proof (for additional details, see [8, Theorem 5.2]).

We start with the existence part of the proof. 
Let us write the problem (3.5) in the equivalent form:

$$
\left\{\begin{array}{l}
\Delta \mathbf{w}_{+}-\mathcal{P} \mathbf{w}_{+}-\nabla p_{+}=\left.\mathfrak{F}\right|_{\Omega_{+}}+\mathcal{J}_{k, \beta, \Omega_{+}}\left(\mathbf{w}_{+}\right) \text {in } \Omega_{+}, \\
\Delta \mathbf{w}_{-}-\alpha \mathbf{w}_{-}-\nabla p_{-}=\left.\mathfrak{F}\right|_{\Omega_{-}} \text {in } \Omega_{-}, \\
\operatorname{Tr}^{+} \mathbf{w}_{+}-T r^{-} \mathbf{w}_{-}=\mathfrak{G}_{0} \text { on } \partial \Omega \\
\mathbf{t}_{\mathcal{P}, \nu}^{+}\left(\mathbf{w}_{+}, p_{+}, \mathfrak{F}_{+}+\mathcal{J}_{k, \beta, \Omega_{+}}\left(\mathbf{w}_{+}\right)\right) \\
\quad-\mathbf{t}_{\alpha, \nu}^{-}\left(\mathbf{w}_{-}, p_{-}, \mathfrak{F}_{-}\right)+\boldsymbol{L} T r^{+} \mathbf{w}_{+}=\mathfrak{H}_{0} \text { on } \partial \Omega,
\end{array}\right.
$$

where $\mathcal{J}_{k, \beta, \Omega_{+}}\left(\mathbf{w}_{+}\right) \in \widetilde{H}^{-1}\left(\Omega_{+}\right)^{3}$ is given by Lemma 2.8 .

Now, we fix $\mathbf{w}_{+} \in H_{\text {div }}^{1}\left(\Omega_{+}\right)^{3}$ and consider the following linear transmission problem for the generalized and classical Brinkman systems with the unknowns $\left(\mathbf{w}_{+}^{0}, p_{+}^{0}, \mathbf{w}_{-}^{0}, p_{-}^{0}\right)$ :

$$
\left\{\begin{array}{l}
\Delta \mathbf{w}_{+}^{0}-\mathcal{P} \mathbf{w}_{+}^{0}-\nabla p_{+}^{0}=\left.\mathfrak{F}\right|_{\Omega_{+}}+\mathcal{J}_{k, \beta, \Omega_{+}}\left(\mathbf{w}_{+}\right) \text {in } \Omega_{+} \\
\Delta \mathbf{w}_{-}^{0}-\alpha \mathbf{w}_{-}^{0}-\nabla p_{-}^{0}=\left.\mathfrak{F}\right|_{\Omega_{-}} \text {in } \Omega_{-} \\
\operatorname{Tr}^{+} \mathbf{w}_{+}^{0}-\operatorname{Tr}^{-} \mathbf{w}_{-}^{0}=\mathfrak{G}_{0} \text { on } \partial \Omega \\
\mathbf{t}_{\mathcal{P}, \nu}^{+}\left(\mathbf{w}_{+}^{0}, p_{+}^{0}, \mathfrak{F}_{+}+\mathcal{J}_{k, \beta, \Omega_{+}}\left(\mathbf{w}_{+}\right)\right) \\
\quad-\mathbf{t}_{\alpha, \nu}^{-}\left(\mathbf{w}_{-}^{0}, p_{-}^{0}, \mathfrak{F}_{-}\right)+\boldsymbol{L} T r^{+} \mathbf{w}_{+}^{0}=\mathfrak{H}_{0} \text { on } \partial \Omega
\end{array}\right.
$$

By applying Theorem 3.1 we deduce that the problem (3.10) has a unique solution $\left(\mathbf{w}_{+}^{0}, p_{+}^{0}, \mathbf{w}_{-}^{0}, p_{-}^{0}\right)$ in $\mathfrak{X}$ given by

$$
\begin{aligned}
& \left(\mathbf{w}_{+}^{0}, p_{+}^{0}, \mathbf{w}_{-}^{0}, p_{-}^{0}\right)=\left(\mathcal{W}_{+}\left(\mathbf{w}_{+}\right), \mathfrak{P}_{+}\left(\mathbf{w}_{+}\right), \mathcal{W}_{-}\left(\mathbf{w}_{+}\right), \mathfrak{P}-\left(\mathbf{w}_{+}\right)\right) \\
& :=\mathcal{T}\left(\mathfrak{F}_{+}\left|\Omega_{+}+\mathcal{J}_{k, \beta, \Omega_{+}}\left(\mathbf{w}_{+}\right)\right|_{\Omega_{+}},\left.\mathfrak{F}_{-}\right|_{\Omega_{-}}, \mathfrak{G}_{0}, \mathfrak{H}_{0}\right) \in \mathfrak{X},
\end{aligned}
$$

where $\mathcal{T}$ is the solution operator introduced in Theorem 3.1.

Note that, for fixed given data $\mathfrak{F}_{ \pm}, \mathfrak{G}_{0}, \mathfrak{H}_{0}$, the nonlinear operators

$$
\mathcal{W}_{ \pm}, \mathfrak{P}_{ \pm}: H_{\mathrm{div}}^{1}\left(\Omega_{+}\right)^{3} \rightarrow \mathfrak{X},
$$

are bounded, in the sense that there exists a constant $d \equiv d\left(\Omega_{+}, \Omega_{-}, \mathcal{P}, \boldsymbol{L}\right)>0$, a constant, such that

$$
\begin{aligned}
\|\left(\mathcal{W}_{+}\left(\mathbf{w}_{+}\right),\right. & \left.\mathfrak{P}_{+}\left(\mathbf{w}_{+}\right), \mathcal{W}_{-}\left(\mathbf{w}_{+}\right), \mathfrak{P}_{-}\left(\mathbf{w}_{+}\right)\right) \|_{\mathfrak{X}} \\
& \leq d\left\|\left(\mathfrak{F}_{+}\left|\Omega_{+}+\mathcal{J}_{k, \beta, \Omega_{+}}\left(\mathbf{u}_{+}\right)\right|_{\Omega_{+}}, \mathfrak{F}_{-} \mid \Omega_{-}, \mathfrak{G}_{0}, \mathfrak{H}_{0}\right)\right\|_{\mathfrak{Y}} \\
& \leq d\left\|\left(\left.\mathfrak{F}_{+}\right|_{\Omega_{+}},\left.\mathfrak{F}_{-}\right|_{\Omega_{-}}, \mathfrak{G}_{0}, \mathfrak{H}_{0}\right)\right\|_{\mathfrak{Y}}+d c_{0}\left\|\mathbf{w}_{+}\right\|_{H^{1}\left(\Omega_{+}\right)^{3}}^{2}
\end{aligned}
$$

for all $\mathbf{w}_{+} \in H_{\text {div }}^{1}\left(\Omega_{+}\right)^{3}$. Indeed, such an inequality is provided by Lemma 2.8 and $c_{0}>0$ is the constant involved in Lemma 2.8.

Next, we rewrite the problem (3.10) in terms of the operators $\mathcal{W}_{ \pm}, \mathfrak{P}_{ \pm}$, as

$$
\left\{\begin{array}{l}
\Delta \mathcal{W}_{+}\left(\mathbf{w}_{+}\right)-\mathcal{P} \mathcal{W}_{+}\left(\mathbf{w}_{+}\right)-\nabla \mathfrak{P}_{+}\left(\mathbf{w}_{+}\right)= \\
\left.\quad \mathfrak{F}\right|_{\Omega_{+}}+\mathcal{J}_{k, \beta, \Omega_{+}}\left(\mathbf{w}_{+}\right) \text {in } \Omega_{+} \\
\Delta \mathcal{W}_{-}\left(\mathbf{w}_{+}\right)-\alpha \mathcal{W}_{-}\left(\mathbf{w}_{+}\right)-\nabla \mathfrak{P}_{+}\left(\mathbf{w}_{+}\right)=\left.\mathfrak{F}\right|_{\Omega_{-}} \text {in } \Omega_{-}, \\
\operatorname{Tr}^{+} \mathcal{W}_{+}\left(\mathbf{w}_{+}\right)-\operatorname{Tr}^{-} \mathcal{W}_{-}\left(\mathbf{w}_{+}\right)=\mathfrak{G}_{0} \text { on } \partial \Omega \\
\mathbf{t}_{\mathcal{P}, \nu}^{+}\left(\mathcal{W}_{+}\left(\mathbf{w}_{+}\right), \mathfrak{P}_{+}\left(\mathbf{w}_{+}\right), \mathfrak{F}_{+}+\mathcal{J}_{k, \beta, \Omega_{+}}\left(\mathbf{w}_{+}\right)\right) \\
\quad-\mathbf{t}_{\alpha, \nu}^{-}\left(\mathcal{W}_{-}\left(\mathbf{w}_{+}\right), \mathfrak{P}_{-}\left(\mathbf{w}_{+}\right), \mathfrak{F}_{-}\right)+\boldsymbol{L} \operatorname{Tr}^{+} \mathcal{W}_{+}\left(\mathbf{w}_{+}\right)=\mathfrak{H}_{0} \text { on } \partial \Omega .
\end{array}\right.
$$


The next step is to show that the nonlinear operator $\mathcal{W}_{+}$has a unique fixed point. If we are able to show this property, then the fixed point $\mathbf{w}_{+} \in H_{\text {div }}^{1}\left(\Omega_{+}\right)^{3}$, together with the fields $\mathbf{w}_{-}=\mathcal{W}_{-}\left(\mathbf{w}_{+}\right)$and with $p_{ \pm}=\mathfrak{P}_{ \pm}\left(\mathbf{w}_{+}\right)$will give a solution of our nonlinear problem (3.9).

Now, we use similar ideas to those in the proof of Theorem 5.2 in [8], to show that $\mathcal{W}_{+}$maps a closed ball $\mathbf{B}_{\lambda}$ to the same closed ball in $H_{\text {div }}^{1}\left(\Omega_{+}\right)^{3}$ and that $\mathcal{W}_{+}$is a contraction on that ball.

We make the following choice of constants

$$
\xi:=\frac{3}{16 c_{0} d^{2}}>0, \quad \lambda:=\frac{1}{4 c_{0} d}>0,
$$

and we introduce the closed ball

$$
\mathbf{B}_{\lambda}:=\left\{\mathbf{v}_{+} \in H_{\text {div }}^{1}\left(\Omega_{+}\right)^{3}:\left\|\mathbf{v}_{+}\right\|_{H^{1}\left(\Omega_{+}\right)^{3}} \leq \lambda\right\} .
$$

We impose the following condition on the given data:

$$
\|\left.\left(\left.\mathfrak{F}_{+}\right|_{\Omega_{+}},\left.\mathfrak{F}_{-}\right|_{\Omega_{-}}, \mathfrak{G}_{0}, \mathfrak{H}_{0}\right)\right|_{\mathfrak{Y}} \leq \xi .
$$

Then, by using relations (3.13), (3.15), (3.16), (3.17), one may show that

$$
\left\|\left(\mathcal{W}_{+}\left(\mathbf{w}_{+}\right), \mathfrak{P}_{+}\left(\mathbf{w}_{+}\right), \mathcal{W}_{-}\left(\mathbf{w}_{+}\right), \mathfrak{P}_{-}\left(\mathbf{w}_{+}\right)\right)\right\|_{\mathfrak{X}} \leq \lambda,
$$

for all $\mathbf{w}_{+} \in \mathbf{B}_{\lambda}$ and hence $\left\|\mathcal{W}_{+}\left(\mathbf{w}_{+}\right)\right\|_{H^{1}\left(\Omega_{+}\right)^{3}} \leq \lambda$ for all $\mathbf{w}_{+} \in \mathbf{B}_{\lambda}$, that is, $\mathcal{W}_{+}$maps the ball $\mathbf{B}_{\lambda}$ to itself.

In order to show that $\mathcal{W}_{+}$is Lipschitz continuous on $\mathbf{B}_{\lambda}$, we fix the given data $\left(\left.\mathfrak{F}_{+}\right|_{\Omega_{+}},\left.\mathfrak{F}_{-}\right|_{\Omega_{-}}, \mathfrak{G}_{0}, \mathfrak{H}_{0}\right)$ and we consider two arbitrary functions $\mathbf{w}_{+}, \mathbf{v}_{+} \in \mathbf{B}_{\lambda}$. Then, we get

$$
\begin{aligned}
& \left\|\mathcal{W}_{+}\left(\mathbf{w}_{+}\right)-\mathcal{W}_{+}\left(\mathbf{v}_{+}\right)\right\|_{H^{1}\left(\Omega_{+}\right)^{3}} \\
\leq & d\left\|\mathcal{J}_{k, \beta, \Omega_{+}}\left(\mathbf{w}_{+}\right)-\mathcal{J}_{k, \beta, \Omega_{+}}\left(\mathbf{v}_{+}\right)\right\|_{\widetilde{H}^{-1}\left(\Omega_{+}\right)^{3}} \\
\leq & d c_{0}\left(\left\|\mathbf{w}_{+}\right\|_{H^{1}\left(\Omega_{+}\right)^{3}}+\left\|\mathbf{v}_{+}\right\|_{H^{1}\left(\Omega_{+}\right)^{3}}\right)\left\|\mathbf{w}_{+}-\mathbf{v}_{+}\right\|_{H^{1}\left(\Omega_{+}\right)^{3}} \\
\leq & 2 d c_{0}\left\|\mathbf{w}_{+}-\mathbf{v}_{+}\right\|_{H^{1}\left(\Omega_{+}\right)^{3}}=\frac{1}{2}\left\|\mathbf{w}_{+}-\mathbf{v}_{+}\right\|_{H^{1}\left(\Omega_{+}\right)^{3}},
\end{aligned}
$$

for all $\mathbf{w}_{+}, \mathbf{v}_{+} \in \mathbf{B}_{\lambda}$, where we have take into account the continuity of the operator $\mathcal{T}$ and inequality (2.12) and the constants $d$ and $c_{0}$ are the same constants as in relation (3.13). Based on the above considerations, we deduce that $\mathcal{W}_{+}: \mathbf{B}_{\lambda} \rightarrow \mathbf{B}_{\lambda}$ is a $\frac{1}{2}$-contraction.

By applying Banach's fixed point theorem we deduce that there is a unique fixed point $\mathbf{w}_{+} \in \mathbf{B}_{\lambda}$ of the operator $\mathcal{W}_{+}$, which, together with the fields given by $\mathbf{w}_{-}=\mathcal{W}_{-}\left(\mathbf{w}_{+}\right)$and $p_{ \pm}=\mathfrak{P}_{ \pm}\left(\mathbf{w}_{+}\right)$, determines a solution of the transmission problem $(3.9)$.

Now, we use the fact that the field $\mathbf{w}_{+} \in \mathbf{B}_{\lambda}$ in order to deduce that

$$
d c_{0}\left\|\mathbf{w}_{+}\right\|_{H^{1}\left(\Omega_{+}\right)^{3}} \leq \frac{1}{4},
$$

and by using inequality (3.13), we obtain

$$
\begin{array}{r}
\left\|\mathbf{w}_{+}\right\|_{H^{1}\left(\Omega_{+}\right)^{3}}+\left\|p_{+}\right\|_{L^{2}\left(\Omega_{+}\right)}+\left\|\mathbf{w}_{-}\right\|_{H^{1}\left(\Omega_{-}\right)^{3}}+\left.\left\|p_{-}\right\|\right|_{\mathfrak{M}\left(\Omega_{-}\right)} \\
\leq d\left\|\left(\mathfrak{F}_{+}\left|\Omega_{+}, \mathfrak{F}_{-}\right|_{\Omega_{-}}, \mathfrak{G}_{0}, \mathfrak{H}_{0}\right)\right\|_{\mathfrak{Y}}+\frac{1}{4}\left\|\mathbf{w}_{+}\right\|_{H^{1}\left(\Omega_{+}\right)^{3}},
\end{array}
$$


and we obtain that

$$
\left\|\mathbf{w}_{+}\right\|_{H^{1}\left(\Omega_{+}\right)^{3}} \leq \frac{4}{3} d\left\|\left(\left.\mathfrak{F}_{+}\right|_{\Omega_{+}},\left.\mathfrak{F}_{-}\right|_{\Omega_{-}}, \mathfrak{G}_{0}, \mathfrak{H}_{0}\right)\right\|_{\mathfrak{Y}} .
$$

By substituting relation (3.21) into (3.20) we obtain the desired estimate (3.8) where $C_{0}=\frac{4}{3} d$.

For other details, we refer to the proof of Theorem 5.2 in [8].

As for the uniqueness part, the ideas are as follows.

Assume that we have two solutions of the transmission problem (3.5), say $\left(\mathbf{w}_{+}^{1}, p_{+}^{1}, \mathbf{w}_{-}^{1}, p_{-}^{1}\right)$ and $\left(\mathbf{w}_{+}^{2}, p_{+}^{2}, \mathbf{w}_{-}^{2}, p_{-}^{2}\right)$. Note that these solutions belong to the space $\mathfrak{X}$ and both satisfy inequality $(3.7)$.

We obtain the linear, homogeneous transmission problem for the classical and generalized Brinkman systems in Lipschitz domains in $\mathbb{R}^{3}$ with the unknowns $\left(\mathcal{W}_{+}\left(\mathbf{w}_{+}^{2}\right)-\mathbf{w}_{+}^{2}, \mathfrak{P}_{+}\left(\mathbf{w}_{+}^{2}\right)-p_{+}^{2}, \mathcal{W}_{-}\left(\mathbf{w}_{+}^{2}\right)-\mathbf{w}_{-}^{2}, \mathfrak{P}_{-}\left(\mathbf{w}_{+}^{2}\right)-p_{-}^{2}\right)$ and Theorem 3.1 shows that this problem has only the trivial solution in $\mathfrak{X}$. It follows that $\mathcal{W}_{+}\left(\mathbf{w}_{+}^{2}\right)-\mathbf{w}_{+}^{2}=0$, that is, $\mathbf{w}_{+}^{2}$ is a fixed point of the nonlinear operator $\mathcal{W}_{+}$. Recall that $\mathcal{W}_{+}: \mathbf{B}_{\lambda} \rightarrow \mathbf{B}_{\lambda}$ is a $\frac{1}{2}$-contractions, and hence, there is a unique fixed point $\mathbf{w}_{+}^{1}$ in $\mathbf{B}_{\lambda}$. Consequently, $\mathbf{w}_{+}^{2}=\mathbf{w}_{+}^{1}, \mathbf{w}_{-}^{2}=\mathbf{w}_{-}^{1}$ and $p_{ \pm}^{2}=p_{ \pm}^{1}$.

This concludes the uniqueness argument.

This concludes the proof.

Remark 3.3. (i) If $k=0$ and $\beta: \Omega_{+} \rightarrow \mathbb{R}_{+}$such that $\beta \in L^{\infty}\left(\Omega_{+}\right)$, then we get the well-posedness result for the nonlinear transmission problem for the generalized Navier-Stokes and Brinkman systems in complementary Lipschitz domains in $\mathbb{R}^{3}$.

(ii) If $k: \Omega_{+} \rightarrow \mathbb{R}_{+}$such that $k \in L^{\infty}\left(\Omega_{+}\right)$and $\beta=0$, then we get the well-posedness result for a semilinear transmission problem for a generalized semilinear DarcyForchheimer-Brinkman system and the Brinkman system in complementary Lipschitz domains in $\mathbb{R}^{3}$.

All these problems are important for their practical applications (see, e.g., [21], [5]).

Acknowledgement. The author acknowledges the support of the Grant PN-III-P4-IDPCE-2016-0036 of the Romanian National Authority for Scientific Research, CNCSUEFISCDI.

\section{References}

[1] Agranovich, M.S., Sobolev Spaces, Their Generalizations and Elliptic Problems in Smooth and Lipschitz Domains, Springer International Publishing, Cham, 2015.

[2] Albişoru, A.F., A layer potential analysis for transmission problems for Brinkman-type systems in Lipschitz domains in $\mathbb{R}^{3}$, Math. Nachr., DOI:10.1002/mana.201800139.

[3] Albişoru, A.F., Transmission-type problems for the generalized Darcy-ForchheimerBrinkman and Stokes systems in complementary Lipschitz domains in $\mathbb{R}^{3}$, Filomat, accepted.

[4] Escauriaza, L., Mitrea, M., Transmission problems and spectral theory for singular integral operators on Lipschitz domains, J. Funct. Anal., 216(2004), 141-171. 
[5] Galdi, G.P., An Introduction to the Mathematical Theory of the Navier-Stokes Equations, Vol. I, II, Springer, Berlin, 1998.

[6] Groşan, T., Kohr, M., Wendland, W.L., Dirichlet problem for a nonlinear generalized Darcy-Forchheimer-Brinkman system in Lipschitz domains, Math. Methods Appl. Sci., 38(2015), 3615-3628.

[7] Hsiao, G.C., Wendland, W.L., Boundary Integral Equations: Variational Methods, Springer, Heidelberg, 2008.

[8] Kohr, M., Lanza de Cristoforis, M., Mikhailov, S.E., Wendland, W.L., Integral potential method for a transmission problem with Lipschitz interface in $\mathbb{R}^{3}$ for the Stokes and Darcy-Forchheimer-Brinkman PDE Systems, Z. Angew. Math. Phys., 67:116, 5(2016), $1-30$.

[9] Kohr, M., Lanza de Cristoforis, M., Wendland, W.L., Boundary value problems of Robin type for the Brinkman and Darcy-Forchheimer-Brinkman systems in Lipschitz domains, J. Math. Fluid Mech., 16(2014), 595-630.

[10] Kohr, M., Lanza de Cristoforis, M., Wendland, W.L., Nonlinear Darcy-ForchheimerBrinkman System with Linear Robin Boundary Conditions in Lipschitz Domains, Complex Analysis and Potential Theory with Applications (T. Aliev Azeroglu, A. Golberg, S. Rogosin eds.), Cambridge Scientific Publishers, 2014, 111-124.

[11] Kohr, M., Lanza de Cristoforis, M., Wendland, W.L., Nonlinear Neumann-transmission problems for Stokes and Brinkman equations on Euclidean Lipschitz domains, Potential Anal., 38(2013), 1123-1171.

[12] Kohr, M., Lanza de Cristoforis, M., Wendland, W.L., On the Robin-transmission boundary value oroblems for the Darcy-Forchheimer-Brinkman and Navier-Stokes systems, J. Math. Fluid Mech., 18(2016), 293-329.

[13] Kohr, M., Lanza de Cristoforis, M., Wendland, W.L., Poisson problems for semilinear Brinkman systems on Lipschitz domains in $\mathbb{R}^{n}$, Z. Angew. Math. Phys., 66(2015), 833864 .

[14] Kohr, M., Mikhailov, S.E., Wendland, W.L., Transmission problems for the NavierStokes and Darcy-Forchheimer-Brinkman systems in Lipschitz domains on compact Riemannian manifolds, J. Math. Fluid Mech., 19(2017), 203-238.

[15] Kohr, M., Wendland, W.L., Variational approach for the Stokes and Navier-Stokes systems with nonsmooth coefficients in Lipschitz domains on compact Riemannian manifolds, Calc. Var. Partial Differential Equations, 57:165, https://doi.org/10.1007/s00526018-1426-7.

[16] Medkova, D., Transmission problem for the Brinkman system, Complex Var. Elliptic Equ., 59(2014), 1664-1678.

[17] Medkova, D., Integral equations method and the transmission problem for the Stokes system, Kragujevac J. Math., 39(2015), 53-71.

[18] Mikhailov, S.E., Traces, extensions and co-normal derivatives for elliptic systems on Lipschitz domains, J. Math. Anal. Appl., 378(2011), 324-342.

[19] Mitrea, D., Mitrea, M., Shi, Q., Variable coefficient transmission problems and singular integral operators on non-smooth manifolds, J. Integral Equations Appl., 18(2006), 361397.

[20] Mitrea, M., Wright, M., Boundary Value Problems for the Stokes System in Arbitrary Lipschitz Domains, Asterisque, 344(2012), viii+241.

[21] Nield, D.A., Bejan, A., Convection in Porous Media, 3rd edn., Springer, New York, 2013. 
Andrei-Florin Albişoru

Babeş-Bolyai University

Faculty of Mathematics and Computer Science

1, Kogălniceanu Street

400084 Cluj-Napoca, Romania

e-mail: florin.albisoru@math.ubbcluj.ro 